\title{
A relation between fidelity and quantum adiabatic evolution
}

\author{
Zhaohui Wei and Mingsheng Ying \\ State Key Laboratory of Intelligent Technology and Systems, \\ Department of Computer Science and Technology, Tsinghua University, Beijing, China, 100084
}

\begin{abstract}
Recently, some quantum algorithms have been implemented by quantum adiabatic evolutions. In this paper, we discuss the accurate relation between the running time and the distance of the initial state and the final state of a kind of quantum adiabatic evolutions. We show that this relation can be generalized to the case of mixed states.
\end{abstract}

PACS numbers: 03.67.Lx, 89.70.+c

Implementing quantum algorithms via quantum adiabatic evolutions is a novel paradigm for the design of quantum algorithms, which was proposed by Farhi et al. [1]. In a quantum adiabatic algorithm, the evolution of the quantum register is governed by a hamiltonian that varies continuously and slowly. At the beginning, the state of the system is the ground state of the initial hamiltonian. If we encode the solution of the algorithm in the ground state of the final hamiltonian and if the hamiltonian of the system evolves slowly enough, the quantum adiabatic theorem guarantees that the final state of the system will differ from the ground state of the final hamiltonian by a negligible amount. Thus after the quantum adiabatic evolution we can get the solution with high probability by measuring the final state. For example, Quantum search algorithm proposed by Grover 2] has been implemented by quantum adiabatic evolution in 3]. Recently, the new paradigm for quantum computation has been tried to solve some other interesting and important problems [4, 5, 6]. For example, T. D. Kieu has proposed a quantum adiabatic algorithm for Hilbert's tenth problem [5], while this problem is known to be mathematically noncomputable.

Usually, after the design of a quantum adiabatic evolution, the estimation of the running time is not easy. In 3], Roland et al. introduced a policy to design a class of quantum local adiabatic evolutions with a performance that can be estimated accurately. Using this policy Roland et al. reproduced quantum search algorithm, which is as good as Grover's algorithm.

For convenience of the readers, we briefly recall the local adiabatic algorithm. Suppose $H_{0}$ and $H_{T}$ are the initial and the final Hamiltonians of the system, we choose them as

$$
H_{0}=I-|\alpha\rangle\langle\alpha|,
$$

and

$$
H_{T}=I-|\beta\rangle\langle\beta|,
$$

where $|\alpha\rangle$ is the initial state of the system and $|\beta\rangle$ is the final state that encodes the solution. Then we let the system vary under the following time dependent Hamiltonian:

$$
H(t)=(1-s) H_{0}+s H_{T},
$$

where $s=s(t)$ is a monotonic function with $s(0)=0$ and $s(T)=1$ ( $T$ is the running time of the evolution). Let $\left|E_{0}, t\right\rangle$ and $\left|E_{1}, t\right\rangle$ be the ground state and the first excited state of the Hamiltonian at time t, and let $E_{0}(t)$ and $E_{1}(t)$ be the corresponding eigenvalues. The adiabatic theorem 7] shows that we have

$$
\left|\left\langle E_{0}, T \mid \psi(T)\right\rangle\right|^{2} \geq 1-\varepsilon^{2},
$$

provided that

$$
\frac{D_{\max }}{g_{\min }^{2}} \leq \varepsilon, \quad 0<\varepsilon \ll 1,
$$

where $g_{\min }$ is the minimum gap between $E_{0}(t)$ and $E_{1}(t)$

$$
g_{\min }=\min _{0 \leq t \leq T}\left[E_{1}(t)-E_{0}(t)\right],
$$

and $D_{\max }$ is a measurement of the evolving rate of the Hamiltonian

$$
D_{\max }=\max _{0 \leq t \leq T}\left|\left\langle\frac{d H}{d t}\right\rangle_{1,0}\right|=\max _{0 \leq t \leq T}\left|\left\langle E_{1}, t\left|\frac{d H}{d t}\right| E_{0}, t\right\rangle\right| .
$$

In the local adiabatic evolution of $\underline{3}$,

$$
|\alpha\rangle=\frac{1}{\sqrt{N}} \sum_{i=1}^{N}|i\rangle,|\beta\rangle=|m\rangle,
$$

where $N$ is the size of the database and $m$ is the solution of the search problem. To evaluate the running time of the adiabatic evolution, Roland and Cerf calculated accurately the gap $g_{\min }$ in Eq. (2) and just estimated the quantity $D_{\max }$ in Eq. (3) using the bound

$$
\left|\left\langle\frac{d H}{d t}\right\rangle_{1,0}\right| \leq\left|\frac{d s}{d t}\right| .
$$

To evaluate the performance of this algorithms, this is enough, because calculating accurately the quantity $D_{\max }$ in (7) can't improve the result much. However, in this paper we will take into account all the related quantities. Later we will find that this will result in a simple and intrinsical relation between the running time of the adiabatic evolution and the distance of the initial and the final states. 
In this paper, we will choose fidelity, one of the most popular distance measures in the literature, as the measure of the hardness to evolve from one state to another using adiabatic evolutions.

The fidelity of states $\rho$ and $\sigma$ is defined to be

$$
F(\rho, \sigma)=\operatorname{tr} \sqrt{\rho^{1 / 2} \sigma \rho^{1 / 2}}
$$

Although fidelity is not a metric, its modified version

$$
A(\rho, \sigma)=\arccos F(\rho, \sigma)
$$

is easily proved to be a metric 11]. Another important metric for the distance between quantum states we will use in this paper is the trace distance defined as

$$
D(\rho, \sigma)=\frac{1}{2} \operatorname{tr}|\rho-\sigma|
$$

Now, we can represent the main result as the following theorem.

Theorem 1 Suppose $|\alpha\rangle$ and $|\beta\rangle$ are two states of a quantum system. We can make the system evolve from the initial state $|\alpha\rangle$ to the final state $|\beta\rangle$ by a quantum adiabatic evolution, if we set the initial Hamiltonian $H_{0}$ and the final Hamiltonian $H_{T}$ of the adiabatic evolution as follows:

$$
\begin{aligned}
& H_{0}=I-|\alpha\rangle\langle\alpha|, \\
& H_{T}=I-|\beta\rangle\langle\beta| .
\end{aligned}
$$

To success with a probability at least $1-\varepsilon^{2}$, the minimal running time that the adiabatic evolution requires is

$$
T(|\alpha\rangle,|\beta\rangle)=\frac{1}{\varepsilon} \cdot \tan (\arccos F(|\alpha\rangle,|\beta\rangle)),
$$

where

$$
F(|\alpha\rangle,|\beta\rangle)=|\langle\alpha \mid \beta\rangle|
$$

is the fidelity between $|\alpha\rangle$ and $|\beta\rangle$.

Proof. Let

$$
H(s)=(1-s)(I-|\alpha\rangle\langle\alpha|)+s(I-|\beta\rangle\langle\beta|),
$$

where $s=s(t)$ is a function of $t$ as described above.

It is not easy to calculate the eigenvalues of $H(s)$ in the computational basis. We use the following orthonormal basis $\{|i\rangle, 1 \leq i \leq N\}$ to eliminate the difficulty:

$$
\begin{gathered}
|1\rangle=|\alpha\rangle, \\
|2\rangle=\frac{1}{c}(|\beta\rangle-\langle\alpha \mid \beta\rangle|\alpha\rangle),
\end{gathered}
$$

where $c=|| \beta\rangle-\langle\alpha \mid \beta\rangle|\alpha\rangle \mid=\sqrt{1-|\langle\alpha \mid \beta\rangle|^{2}}$. We don't need to care about $\left|\alpha_{i}\right\rangle$ for $i=3,4, \ldots, N$. Then we have

$$
|\beta\rangle=c|2\rangle+\langle\alpha \mid \beta\rangle|1\rangle \text {. }
$$

Now it is not difficult to check that, in the new orthonormal basis, $H(s)$ has a form of

$$
H(s)=\left(\begin{array}{ccc}
-s|\langle\alpha \mid \beta\rangle|^{2}+s & -s c\langle\alpha \mid \beta\rangle & \\
-s c\langle\alpha \mid \beta\rangle^{*} & -s c^{2}+1 & \\
& & I_{(N-2) \times(N-2)}
\end{array}\right),
$$

where the empty spaces of the matrix are all zeroes. Letting $a=|\langle\alpha \mid \beta\rangle|$, it is easy to get the two lowest eigenvalues of $H(s)$

$$
E_{i}(t)=\frac{1}{2}\left(1 \pm \sqrt{1-4\left(1-a^{2}\right) s(1-s)}\right), i=0,1
$$

and two corresponding eigenvectors

$$
\left|E_{i}, t\right\rangle=\frac{1}{\sqrt{1+y_{i}^{2}}}\left(|1\rangle+y_{i}|2\rangle\right), i=0,1
$$

where

$$
y_{i}=\frac{\sqrt{1-a^{2}}}{a}-\frac{E_{i}(t)}{s a \sqrt{1-a^{2}}}(s \neq 0) .
$$

Thus, we get $g(s)$ :

$$
g(s)=\sqrt{1-4\left(1-a^{2}\right) s(1-s)} .
$$

On the other hand, it is easy to kown

$$
\frac{d H}{d s}=H_{T}-H_{0}=\frac{H(s)-H_{0}}{s} \quad(s \neq 0) .
$$

Because $\left|E_{0}, t\right\rangle$ and $\left|E_{1}, t\right\rangle$ are eigenvectors of $H(s)$, we have

$$
\left\langle E_{0}, t \mid E_{1}, t\right\rangle=0
$$

and

$$
\left\langle E_{0}, t|H(s)| E_{1}, t\right\rangle=0 .
$$

Then it can be shown that

$\left|\left\langle\frac{d H}{d s}\right\rangle_{0,1}\right|=\left|\left\langle E_{0}, t\left|\frac{H(s)-H_{0}}{s}\right| E_{1}, t\right\rangle\right|=\left|\frac{\left\langle E_{0}, t \mid 1\right\rangle\left\langle 1 \mid E_{1}, t\right\rangle}{s}\right|$.

So

$\left|\left\langle\frac{d H}{d t}\right\rangle_{0,1}\right|=\left|\frac{d s}{d t}\right| \cdot\left|\left\langle\frac{d H}{d s}\right\rangle_{0,1}\right|=\left|\frac{d s}{d t}\right| \cdot \frac{1}{s \sqrt{\left(1+y_{0}^{2}\right)\left(1+y_{1}^{2}\right)}}$.

Substituting Eq.(21) into Eq.(27) we have

$$
\left|\left\langle\frac{d H}{d t}\right\rangle_{0,1}\right|=\left|\frac{d s}{d t}\right| \cdot \frac{a \sqrt{1-a^{2}}}{\sqrt{1-4\left(1-a^{2}\right) s(1-s)}} .
$$


In a local adiabatic evolution [3], the adiabaticity condition (5) must be satisfied at any instant of time $t$,

$$
\left|\frac{d s}{d t}\right| \cdot \frac{a \sqrt{1-a^{2}}}{\sqrt{1-4\left(1-a^{2}\right) s(1-s)}} \leq \varepsilon\left(1-4\left(1-a^{2}\right) s(1-s)\right) .
$$

To make the evolution as fast as possible, we can let $s(t)$ satisfy the equation

$$
\frac{d s}{d t}=\varepsilon \frac{\left(1-4\left(1-a^{2}\right) s(1-s)\right)^{\frac{3}{2}}}{a \sqrt{1-a^{2}}} .
$$

By integration, we can get the lower bound of the running time of the whole evolution

$$
T(|\alpha\rangle,|\beta\rangle)=\frac{1}{\varepsilon} \cdot \frac{\sqrt{1-a^{2}}}{a}=\frac{1}{\varepsilon} \cdot \tan (\arccos F(|\alpha\rangle,|\beta\rangle)) .
$$

That completes the proof of this theorem.

In fact, it is interesting to notice that we can rewrite the relation above as

$$
T(|\alpha\rangle,|\beta\rangle)=\frac{1}{\varepsilon} \cdot \frac{D(|\alpha\rangle,|\beta\rangle)}{F(|\alpha\rangle,|\beta\rangle)},
$$

where $D(|\alpha\rangle,|\beta\rangle)$ is the trace distance between $|\alpha\rangle$ and $|\beta\rangle$.

In [3], the fidelity between the initial state and the final state of the local quantum adiabatic evolution is $\frac{1}{\sqrt{N}}$.

According to Theorem 1 the running time is $O(\sqrt{N})$. This is consistent with the result of 3 ].

Similarly, In 8 S. Das et al. implement Deutsch's algorithm 9, 10 by an adiabatic evolution of the form discussed in Theorem 1. In that work, if the system has $n$ qubits, $|\alpha\rangle$ and $|\beta\rangle$ will be $\left(N=2^{n}\right)$

$$
\begin{gathered}
|\alpha\rangle=\frac{1}{\sqrt{N}} \sum_{i=0}^{N-1}|i\rangle, \\
|\beta\rangle=\mu|0\rangle+\frac{\nu}{\sqrt{N-1}} \sum_{i=1}^{N-1}|k\rangle,
\end{gathered}
$$

with

$$
\begin{gathered}
\mu=\frac{1}{N}\left|\sum_{x \in\{0,1\}^{n}}(-1)^{f(x)}\right|, \\
\nu=1-\mu .
\end{gathered}
$$

Here, the function $f:\{0,1\}^{n} \rightarrow\{0,1\}$ is either constant (i.e., all outputs are identical) or balanced (i.e., has an equal number of 0's and 1's as outputs), and our task is to decide whether it is constant or not. It is not difficult to know that in this case

$$
F(|\alpha\rangle,|\beta\rangle)=|\langle\alpha \mid \beta\rangle|=\frac{1}{\sqrt{N}} \text { or } \sqrt{1-\frac{1}{N}} .
$$

To make the algorithm success, we must let the running time of the adiabatic evolution be long enough. So the minimal running time should be $O(\sqrt{N})$. This result is consistent with 8].

Let's try to explain the meaning of the theorem. As we know, $\arccos (F(\rho, \sigma))$ measures the distance of two quantum states $\rho$ and $\sigma$ [1]. Quantum adiabatic evolution, On the other hand, changes the state of a quantum system from the initial state $|\alpha\rangle$ to the final state $|\beta\rangle$. Our theorem says that if the precision of the evolution is fixed, the minimal running time $T(|\alpha\rangle,|\beta\rangle)$ will be direct proportional to the tangent of $\arccos (F(|\alpha\rangle,|\beta\rangle))$. The smaller the distance of the two states is, the shorter the running time of the adiabatic evolution will be. This is consistent with our intuition. However, we should notice that as the fidelity becomes smaller, the running time will increase very quickly. Fore example, when $F(|\alpha\rangle,|\beta\rangle)$ is 0.5 , the running time $T(|\alpha\rangle,|\beta\rangle)$ is $\frac{\sqrt{3}}{\varepsilon}$. While as $F(|\alpha\rangle,|\beta\rangle)$ tends to 0 , the running time tends to infinite.

In the quantum adiabatic evolution, the initial and the final states are pure. Using Uhlmann's theorem [11] we can generalize the relation to the case of mixed states. Suppose $\rho$ and $\sigma$ are two states of a quantum system $A$. Let $B$ is another system and $A$ is a part of $B$. Suppose an adiabatic evolution makes the state of $B$ evolve from $|\psi\rangle$ to $|\varphi\rangle$ and in the same evolution the state of $A$ evolves from $\rho$ to $\sigma$. We may ask - is there any relation between the running time of the adiabatic evolution and the fidelity of $\rho$ and $\sigma$ ? We say yes by the following theorem.

Theorem 2 Suppose $\rho$ and $\sigma$ are two mixed states, and let

$$
T(\rho, \sigma)=\min _{|\psi\rangle,|\varphi\rangle} T(|\psi\rangle,|\varphi\rangle)
$$

where $|\psi\rangle$ is any purification of $\rho$ and and $|\varphi\rangle$ for $\sigma$. Then we have

$$
T(\rho, \sigma)=\frac{1}{\varepsilon} \cdot \tan (\arccos F(\rho, \sigma)),
$$

where $\varepsilon$ is the precision of the evolution.

Proof.

$$
\begin{aligned}
T(\rho, \sigma) & =\min _{|\psi\rangle,|\varphi\rangle} T(|\psi\rangle,|\varphi\rangle) \\
& =\min _{|\psi\rangle,|\varphi\rangle} \frac{1}{\varepsilon} \cdot \tan (\arccos F(|\psi\rangle,|\varphi\rangle)) \\
& =\frac{1}{\varepsilon} \cdot \tan \left(\arccos \left(\max _{|\psi\rangle,|\varphi\rangle} F(|\psi\rangle,|\varphi\rangle)\right)\right) .
\end{aligned}
$$

Applying Uhlmann's theorem [1] to the last equation, we can get

$$
T(\rho, \sigma)=\frac{1}{\varepsilon} \cdot \tan (\arccos F(\rho, \sigma)) .
$$


In conclusion, we have shown the accurate relation between the distance of the initial and the final states and the running time of a class of quantum adiabatic evolution applied in 3]. We have pointed out that via this relation it is convenient to estimate the running times of some adiabatic algorithms. Furthermore, this relation can be generalized to the case of mixed states. This relation maybe can help to design quantum algorithms.

We would like to thank Ji Zhengfeng for useful discussions.
[1] E. Farhi, J. Goldstone, S. Gutmann, and M. Sipser, eprint quant-ph/0001106

[2] L. K. Grover, Phys. Rev. Lett 79, 325(1997).

[3] J. Roland and N. J. Cerf, Phys. Rev. A 65, 042308(2002).

[4] Tad Hogg, Phys. Rev. A 67, 022314(2003).

[5] T. D. Kieu, e-print quant-ph/0110136

[6] E. Farhi et al. e-print quant-ph/0104129

[7] L. I. Schiff, Quantum Mechanics (McGraw-Hill, Singapore, 1955).
[8] S. Das, R. Kobes, G. Kunstatter, Phys. Rev. A 65, 062310(2002).

[9] D. Deutsch, Proc. R. Soc. London, Ser, A 400, 97 (1985).

[10] D. Deutsch and R. Jozsa, Proc. R. Soc. London, Ser, A 439, 553 (1992).

[11] Michael A. Nielsen, Isaac L. Chuang Quantum Computation and Quantum Information, Cambridge University Press, 2000 